CONGENITAL HEART DISEASE

\title{
Long term cardiac follow up of severe twin to twin transfusion syndrome after intrauterine laser coagulation
}

\author{
U Herberg, W Gross, P Bartmann, C S Banek, K Hecher, J Brever
}

Heart 2006;92:95-100. doi: 10.1136/hrt.2004.057497

See end of article for authors' affiliations

Correspondence to: Dr Ulrike Herberg, Division of Paediatric Cardiology, Children's Hospital, University of Bonn, Adenauerallee 119 53113 Bonn, Germany; Ulrike.Herberg@ ukb.uni-bonn.de

Accepted 17 March 2005 Published Online First 6 April 2005

Objective: To assess long term changes in cardiac morphology and function in survivors of severe twin to twin transfusion syndrome (TTS) after intrauterine laser coagulation of placental anastomoses.

Design: Prospective follow up of fetuses with severe TTS treated by laser coagulation of intrauterine placental anastomoses. Fetal echocardiography and Doppler studies of feto-placental haemodynamic function were performed at the time of laser coagulation (median gestational age of 21.7 weeks). Postnatal cardiac follow up included a detailed echocardiographic study of systolic and diastolic cardiac function at a median age of 21.1 months.

Setting: Paediatric cardiology unit.

Patients: 89 survivors from 73 consecutive pregnancies with severe TTTS.

Results: Before laser treatment, 28 of 51 (54.9\%) recipient twins had typical signs of cardiac dysfunction due to volume overload and 9 of $38(23.7 \%)$ donors had absent or reversed end diastolic flow in the umbilical artery. Echocardiography was normal in $87.6 \%$ of the survivors (34 of 38 donors, 44 of 51 recipients). The prevalence of congenital heart disease and particularly of pulmonary stenosis, which was recorded only in recipients, was increased in comparison with the general population (congenital heart disease, 10 of $89(11.2 \%) \vee 0.3 \%$; pulmonary stenosis, 4 of 51 (7.8\%) v $0.03 \%)$. Findings before laser treatment were not correlated with the development of structural heart disease.

Conclusions: Despite the high rate and severity of prenatal cardiac overload in recipients, the majority of cases of TTTS are normalised after laser treatment. However, given the increased prevalence of congenital heart disease and in particular pulmonary stenosis, intrauterine and postnatal follow up is warranted.

$\mathrm{T}$ win to twin transfusion syndrome (TTTS) is a severe complication of monochorionic twin pregnancies. It carries a high risk of fetal death if left untreated (80$100 \%$ ) and a high perinatal morbidity and mortality. ${ }^{12}$ In TTTS, genetically identical twins are exposed to different haemodynamic conditions and environmental factors. Placental vascular anastomoses provide the anatomical basis for the unbalanced intertwin transfusion from donor to recipient. $^{2-4}$ In the hypervolaemic recipient, cardiomegaly, biventricular hypertrophy, and tricuspid and mitral regurgitation precede the development of more severe cardiac dysfunction and may result in fetal hydrops as the end stage of intrauterine heart failure. ${ }^{56}$ Cardiac dysfunction progresses with increasing gestational age. ${ }^{78}$ In addition, various types of cardiac defects predominantly affecting the right ventricle and pulmonary artery have been reported. ${ }^{5-9}$ These include muscular right ventricular outflow obstruction, valvar pulmonary stenosis and atresia, and left ventricular hypertrophic non-obstructive and obstructive cardiomyopathy. In contrast, the hypovolaemic donor twin shows little cardiac pathology on fetal echocardiography but does manifest increased afterload due to raised placental resistance, as well as evidence of poor renal perfusion.

The two alternative treatments are serial amnioreduction and laser coagulation of placental vascular anastomoses. ${ }^{10}$ Amniocentesis reduces the volume and pressure of the amniotic fluid without altering the anatomical substrate. Consequently, volume overload and cardiac dysfunction continue. Interruption of intertwin vascular anastomoses by laser coagulation offers a causal treatment. The volume load is normalised in the recipient and, after a short period of acute volume overload, the donor fetus's Doppler indices are normal. ${ }^{11-13}$ However, postnatal discordance of arterial distensibility, seen in successfully treated survivors, ${ }^{14}$ provides evidence of the altered vascular programming in the developing heart. The effect of longstanding, differing haemodynamic loading conditions on the developing heart has been examined prenatally. Postnatal follow up studies of survivors after serial amniocentesis have been restricted to the neonatal period.

To exclude progression and ensure long term resolution of haemodynamic abnormalities, we studied the outcome of all survivors from a large well defined cohort of consecutive pregnancies with severe TTTS treated by laser coagulation.

\section{PATIENTS AND METHODS}

\section{Study population}

This was a prospective study of 89 survivors from 73 consecutive twin pregnancies (146 fetuses) after prenatal endoscopic laser treatment of severe TTTS. Hecher et $a l^{10}$ reported the survival rate and fetal outcome of this cohort. In all cases, severe TTTS was diagnosed prenatally, fulfilling the following criteria: gestational age $<25.0$ weeks, single monochorionic placenta with massive polyhydramnios of the recipient, and severe oligohydramnios or anhydramnios in the donor twin.

In all pregnancies, endoscopic laser coagulation of the vascular placental anastomoses was performed at a median gestational age of 21.7 weeks (range 17.7-25.0 weeks) at the Department of Prenatal Diagnosis and Therapy, Barmbek Hospital, Hamburg, Germany. Of the 51 fetuses that died in

Abbreviations: $\mathrm{A}$, late flow velocity; $\mathrm{Cl}$, confidence interval; $\mathrm{E}$, early flow velocity; TTS, twin to twin transfusion syndrome 
utero or through miscarriage, only one recipient was seen to have a pulmonary stenosis on prenatal echocardiography. Patients attended their local referring hospital for further sonographic follow up and delivery. The median gestational age at delivery was 33.7 weeks (range 24.9-40.3 weeks). None of the six neonatal deaths was related to cardiac disease.

All 89 surviving infants were included in a neurodevelopmental ${ }^{15}$ and echocardiographic follow up study. The median age of the 51 recipients and 38 donors at examination was 21.5 months (range 15.5-45 months). Eighty two of the 89 surviving infants underwent detailed echocardiography. Of the remaining seven survivors, four had normal echocardiographic findings in the neonatal period and all seven patients were found to have no murmur on clinical examination. Informed consent was obtained from all parents.

\section{Investigations}

Prenatally, all fetuses underwent detailed fetal echocardiography and Doppler sonography shortly before and after laser coagulation. Holosystolic tricuspid regurgitation and mitral regurgitation of more than half of the systole, hydrops (skin oedema and ascites, and pericardial or pleural effusion), and structural cardiac disease were assessed in both twins. Umbilical artery velocimetry was recorded and classified as positive, absent, or reversed end diastolic flow; and ductus venosus waveforms were classified according to positive, absent, or reversed flow during atrial contraction. Absent or reversed end diastolic flow in the umbilical artery is a sign of increased afterload due to increased placental resistance; a pathological ductus venosus waveform is an indicator for increased right ventricular end diastolic pressure. The results of the Doppler studies have previously been reported as part of a larger study. ${ }^{12}$

At the postnatal examination, parents were interviewed with regard to postnatal cardiac abnormalities and the postnatal course of both twins. The patient files were reviewed for all patients who were found to have abnormal cardiac findings or a pathological ECG or echocardiography on postnatal examination.

A 12 lead ECG was recorded (CS 100; Schiller AG, Baar, Switzerland). Blood pressure, recorded on the right arm, was obtained in a supine position. ${ }^{16}$ After the physical examination, two experienced paediatric cardiologists (WG, UH) performed complete echocardiography, including M mode, two dimensional echocardiography, continuous and pulsed wave Doppler, and colour Doppler echocardiography (Vingmed 800,5 MHz and 3.5 MHz transducer; Vingmed, Horten, Norway). Quantitative M mode data were recorded over three cardiac cycles and the mean values of right and left ventricular end diastolic and end systolic diameter, thickness of the interventricular septum and left ventricular posterior wall, and left atrial and aortic dimension and fractional shortening of the left ventricle were obtained. The results were compared with normal values for Central European children. ${ }^{17}$ To assess the diastolic ventricular function of both the right and left ventricles, pulsed wave Doppler measurements at the mitral valve, pulmonary vein, tricuspid valve, and hepatic vein were determined ${ }^{18}$ and compared with age related normal values from the literature. ${ }^{19-25}$ Variables measured at the mitral and tricuspid valve inlets were the early (E) and late (A) flow velocities and their ratio (E:A), E wave deceleration time, the velocity-time integral of the $\mathrm{E}$ and A waves, the ratio of the velocity-time integrals of $\mathrm{E}$ and A, and the duration of the A wave. At the pulmonary vein, the variables measured were the peak flow velocities during systole and diastole, and the peak flow velocity of reversed flow during atrial contraction, as well as its duration. Sites of Doppler investigation were the orifice of the right upper pulmonary vein in the four chamber view and the mitral and tricuspid valve inflow at the leaflet tip.

Standard echocardiographic assessments of the cardiac anatomy and Doppler velocimetry were also obtained. The presence of valve regurgitation was determined by colour Doppler. Right and left ventricular outflow tract velocities, as well as the flow across the pulmonary and aortic valves, were recorded. Pulmonary valve stenosis was classified as mild (maximum pressure gradient $25-49 \mathrm{~mm} \mathrm{Hg}$ ), moderate (maximum pressure gradient $50-79 \mathrm{~mm} \mathrm{Hg}$ ), or severe (maximum pressure gradient $>80 \mathrm{~mm} \mathrm{Hg}$ or equal to systemic blood pressure).

An existing atrial septal defect was classified as medium sized if the defect had a maximum diameter $\geqslant 6 \mathrm{~mm}$, the ratio of right ventricular to left ventricular diastolic dimensions was increased, and the ECG showed right ventricular hypertrophy; and as small if there was no sign of right ventricular hypertrophy on echocardiography and the size of the defect was between 3-6 $\mathrm{mm}$. Interatrial left to right shunts with a diameter of $<3 \mathrm{~mm}$ were defined as persistent foramen ovale. ${ }^{26}$ Persistent foramen ovale is said to be present in more than $10 \%$ of people depending on the age of the cohort and study design ${ }^{27}$ and are considered to be a nonpathological cardiac finding.

\section{Statistical analysis}

The binominal distribution and Fisher's exact test for the two way contingency tables were used for statistical analysis. For Fisher's exact test, $\mathrm{p}<0.05$ was considered to indicate significance. For statistical analysis, cardiac dimensions were transformed into z scores for a given body surface area.

\section{RESULTS}

Congenital heart disease was not found in 78 survivors $(87.6 \%)$ on physical examination, ECG, and conventional echocardiography. One child had pulmonary hypertension secondary to bronchopulmonary dysplasia. The remaining 10 infants had structural heart disease.

\section{Structural heart disease in TTTS}

In surviving twins, the prevalence of congenital heart disease (table 1) was increased (10 of 89 (11.2\%), 95\% confidence interval (CI) 5.5 to 19.7) in comparison with that of the normal population $(0.29-0.56 \%) .{ }^{28} 29$

Pulmonary stenosis was recorded only in recipients (four of $51(7.84 \%), 95 \%$ CI 2.2 to 18.9$)$ and was more common than in the general population (prevalence $0.033-0.038 \%$ ). Two recipients had severe pulmonary valve stenosis, one of whom required balloon dilatation immediately postnatally and one at the age of 4 months. One recipient had a moderate valvar and another one a minimal supravalvar pulmonary stenosis.

Medium sized and small atrial septal defects were found in the same frequency in both the recipient and donor cohorts. Compared with the normal population, our study group had an increased number of medium sized atrial septal defects (2.3\%, 95\% CI 0.3 to 7.9 ; normal prevalence $0.023-0.032 \%)$.

\section{Correlation between prenatal and postnatal cardiac findings}

Overall, 28 of the 51 (55\%) recipient twins had pathological findings prenatally. The most common abnormalities were cardiac chamber enlargement and hypertrophy, holosystolic tricuspid and mitral valve regurgitation, and pathological Doppler velocities of the ductus venosus as an indicator for increased right ventricular end diastolic pressure. Nine of the 38 donors exhibited absent or reversed end diastolic blood flow in the umbilical arteries. There was no significant difference in the prevalence of postnatal congenital heart 
Table 1 Prevalence of congenital heart disease in survivors after twin to twin transfusion syndrome in the study cohort compared with the prevalence at birth reported by the Baltimore-Washington infant study $(\mathrm{WIS})^{28}$ and Wren et a ${ }^{29}$

\begin{tabular}{|c|c|c|c|c|c|}
\hline \multirow[b]{2}{*}{ Postnatal examination } & \multirow{2}{*}{$\begin{array}{l}\text { All } \\
(n=89)\end{array}$} & \multirow{2}{*}{$\begin{array}{l}\text { Recipients } \\
(n=51)\end{array}$} & \multirow{2}{*}{$\begin{array}{l}\text { Donors } \\
(n=38)\end{array}$} & \multicolumn{2}{|c|}{$\begin{array}{l}\text { Prevalence per } 100 \text { live } \\
\text { births }\end{array}$} \\
\hline & & & & $\mathrm{WIS}^{28}$ & Wren $^{29}$ \\
\hline Normal & $78(87.6 \%)$ & $44(86.3 \%)$ & $34(89.5 \%)$ & & \\
\hline Acquired pulmonary HT & 1 & 0 & 1 & & \\
\hline Congenital heart disease & $10(11.2 \%)$ & $7(13.7 \%)$ & $3(7.89 \%)$ & 0.49 & 0.559 \\
\hline $95 \% \mathrm{Cl}$ & 5.52 to 19.7 & 5.70 to 26.2 & 1.66 to 9.25 & & \\
\hline Pulmonary stenosis & $4(4.49 \%)$ & $4(7.84 \%)$ & $0(0)$ & 0.038 & 0.033 \\
\hline $95 \% \mathrm{Cl}$ & 1.24 to 11.1 & 2.18 to 18.9 & 0 to 9.25 & & \\
\hline ASD (all sizes) & $5(5.62 \%)$ & $2(3.92 \%)$ & $3(7.89 \%)$ & & \\
\hline $95 \% \mathrm{Cl}$ & 1.86 to 12.6 & 0.48 to 13.5 & 0.48 to 13.5 & & \\
\hline Medium sized ASD & $2(2.25 \%)$ & $1(1.96 \%)$ & $1(2.63 \%)$ & 0.032 & 0.023 \\
\hline $95 \% \mathrm{Cl}$ & 0.27 to 7.88 & 0.05 to 10.4 & 0.07 to 13.8 & & \\
\hline Ventricular septal defect & $1(1.12 \%)$ & $1(1.96 \%)$ & $0(0)$ & 0.146 & 0.188 \\
\hline $95 \% \mathrm{Cl}$ & 0.03 to 6.10 & 0.05 to 10.4 & 0.0 to 9.25 & & \\
\hline
\end{tabular}

disease between fetuses with and without abnormal cardiac findings before laser coagulation (table 2).

\section{Prenatal findings in pulmonary stenosis}

Only recipients had pulmonary stenosis; therefore, prenatal findings were reviewed separately. The occurrence of pulmonary stenosis did not differ between fetuses with normal and those with abnormal cardiac findings before laser coagulation. Table 3 shows the prenatal and postnatal findings of all children with pulmonary stenosis.

\section{Postnatal myocardial function}

In children without structural heart disease, cardiac dimensions and systolic function were within the normal range at follow up (fig 1). The right ventricular diameters were normal or less than normal in both the recipient and donor without intertwin discordance.

The complete evaluation of all diastolic parameters necessitated a prolonged and standardised investigation. The young children did not always tolerate this. Left and right ventricular inflow parameters were recorded in all except six survivors, but reliable Doppler velocities of the pulmonary vein were acquired for 29 of 51 (56.8\%) recipients and 18 of $38(47.3 \%)$ donors. All donors and recipients with no heart disease had normal diastolic myocardial function (data not shown). Even among recipients with severe prenatal cardiac insufficiency, systolic and diastolic variables were within the normal range.

\section{DISCUSSION}

This long term follow up study of surviving twins after prenatal laser coagulation of severe TTTS has two important results. Firstly, $87 \%$ of the survivors had a normal cardiac examination. Despite the high rate and severity of prenatal cardiac impairment in recipients, systolic or diastolic ventricular function completely normalises over the long term. This illustrates the remarkable capacity of the fetal heart for ventricular remodelling.

Secondly, the rate of pulmonary stenosis and atrial septal defects in surviving twins is increased. Pulmonary stenosis is recorded only in recipients. Possible mechanisms for this are discussed below.

Previous studies have reported varying prevalences and spectrum of heart disease in TTTS. ${ }^{2-9} 3031$ Postnatal studies have focused on complications in the neonatal period. Follow up beyond the neonatal period has been done in small study cohorts $^{7}$ and restricted to survivors with structural heart disease. However, more than half of the recipient twins in severe TTTS exhibit various stages of volume overload and congestive heart failure antenatally, and haemodynamic changes of the donor twins include hypovolaemia and increased afterload.

Diastolic dysfunction precedes systolic dysfunction and the study of the diastolic inflow is a more sensible parameter to determine ventricular performance.

The typical pattern of diastolic dysfunction has been described in children with cardiomyopathy resulting from increased volume or pressure load of either the right or the

\begin{tabular}{|c|c|c|c|c|c|}
\hline \multirow[b]{2}{*}{ Abnormal finding } & \multicolumn{2}{|l|}{ Present } & \multicolumn{2}{|l|}{ Absent } & \multirow[b]{2}{*}{$\mathrm{p}$ Value } \\
\hline & Prenatally & Postnatally & Prenatally & Postnatally & \\
\hline \multicolumn{6}{|l|}{ Recipients } \\
\hline DVnegA & 16 & $3(18.8 \%)$ & 35 & 4 (11.4\%) & 0.66 \\
\hline TR & 26 & $5(19.2 \%)$ & 25 & $2(8.0 \%)$ & 0.42 \\
\hline MR & 7 & $2(28.6 \%)$ & 44 & 5 (11.4\%) & 0.24 \\
\hline Hydrops & 3 & 0 & 48 & $7(14.6 \%)$ & 1.00 \\
\hline \multicolumn{6}{|l|}{ Donors } \\
\hline UAEDFneg & 9 & 1 (11.2\%) & 29 & $2(6.9 \%)$ & 1.00 \\
\hline
\end{tabular}




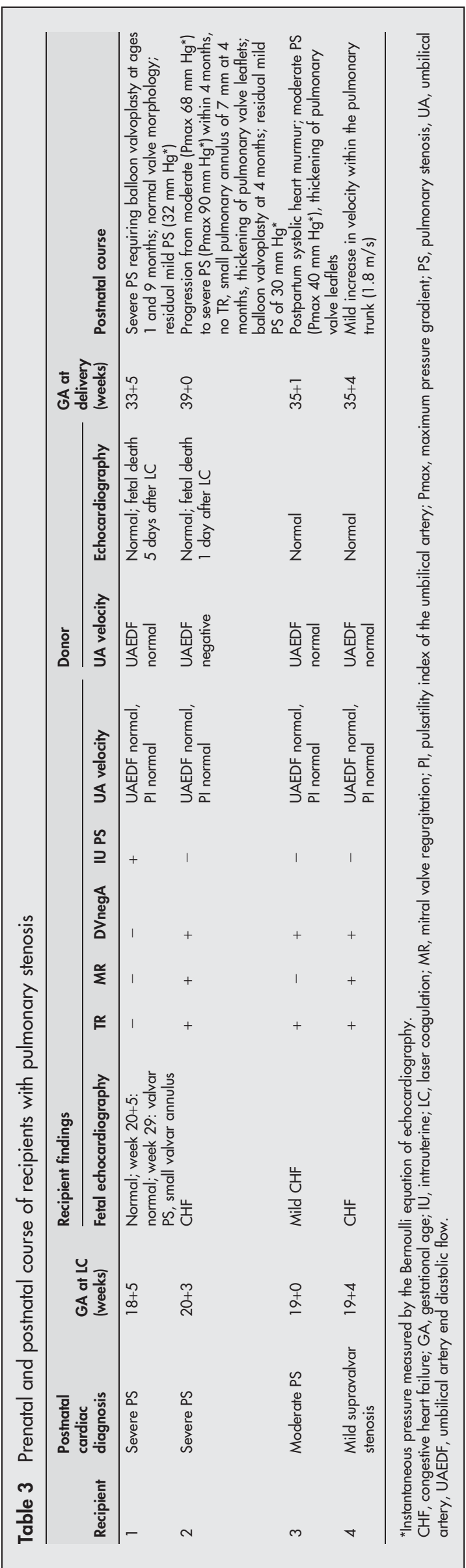

left ventricle. ${ }^{18} 3233$ After relief of the pressure load, or extracardiac or intracardiac shunting, the systolic and diastolic indices in successfully treated patients improve and, over the long term, approach values found in normal subjects. ${ }^{33}$ It is important to note that these parameters are sensitive to loading conditions, heart rate, and age and can vary significantly with inspiration and expiration. ${ }^{18} 192132$ Standardised investigation parameters are difficult to obtain in children below 3 years of age, resulting in highly variable results. This may be why normal values for this age group have been recorded only for small study cohorts, if at all. We were able to show that, at least beyond 15 months of age, even severe prenatal cardiac disease regresses, leaving no symptoms of systolic or diastolic dysfunction. However, we were not able to investigate the exact time point of the normalisation of ventricular function because prenatal and neonatal follow up after laser coagulation was done at the referring hospitals. Longitudinal observations of a small cohort of twins treated with intrauterine amniocentesis found that ventricular hypertrophy, contractility, and atrioventricular valve regurgitation gradually regressed and returned to normal within 40 days to six months after birth. ${ }^{7}$

\section{Congenital heart disease}

Previous studies have reported an increased prevalence of structural heart disease in TTTS. Twins are known to have more congenital malformations. However, even in monochorionic twins, in more than $90 \%$ of cases only one of the twins is affected. ${ }^{34}$ In a prospective study, Karatza et al ${ }^{9}$ compared monochorionic diamniotic pregnancies with and without TTTS (uncomplicated monochorionic diamniotic pregnancies without circulatory imbalance and haemodynamic impairment). They showed that the overall prevalence of congenital heart disease in monochorionic diamniotic twins was increased above that in singletons $16.9 \%$ in TTTS and $2.3 \%$ in uncomplicated monochorionic diamniotic twins compared with $0.56 \%$ in singletons). The increased prevalence of congenital heart disease in fetuses with TTTS was related to the significantly increased number of recipients with congenital heart disease (11.9\%). This is in accordance with the prevalence of $13.7 \%$ found in our study.

The mechanism for the increased rate of congenital heart disease in monochorionic twins is unknown. The monozygotic twinning process itself may increase the incidence of congenital heart disease, with unequal postzygotic division of the inner cell mass being responsible for the discordant cardiovascular anatomy. Another explanation may be the phenotypic variability of the same genome. ${ }^{35}$ Even genetic discordances may result from variations in gene expression secondary to postzygotic mutation, parental imprinting effects, asymmetric $\mathrm{X}$ inactivation, and DNA methylation, ${ }^{36}$ or possibly even genetic intertwin differences caused by new mutations.

Additionally, circulatory imbalance in chronic TTTS may contribute to the development of pulmonary stenosis. Blood flow appears to be crucial to the development and growth of the cardiac chambers, valves, and arteries. ${ }^{14} 37$ Abnormal blood flow may lead to abnormal growth of cardiac and vascular structures. In recipients, a possible mechanism for this is severe and longstanding heart failure caused by chronic right ventricular volume overload, or muscular hypertrophy with severe outflow tract obstruction resulting in diminished antegrade flow with diminished growth of the right ventricular outflow tract and pulmonary artery. Additional environmental factors, such as the release of vasoactive peptides or growth factors, may contribute to the development of haemodynamic imbalance and cardiac disease. $^{931} 38$ 

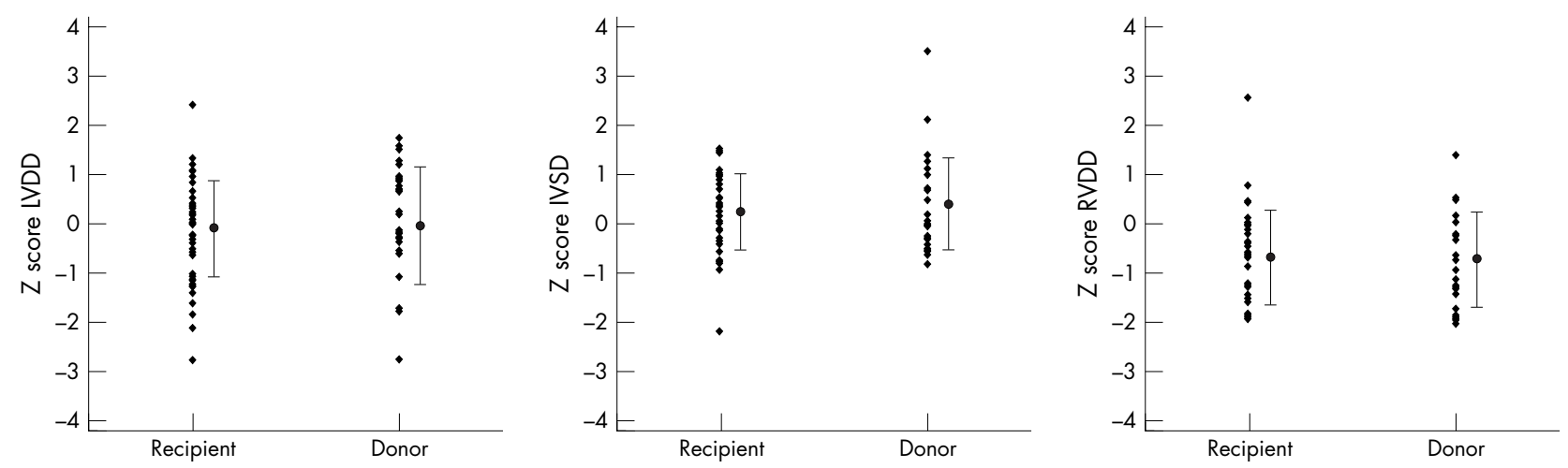

Figure 1 End diastolic dimensions of the left ventricle (LVDD), interventricular septum (IVSD), and right ventricle (RVDD) beyond the age of 15 months in survivors of severe twin to twin transfusion syndrome. Cardiac dimensions are transformed into $z$ scores for a given body surface. There are no intertwin differences.

\section{Effect of laser coagulation}

This is the first cardiological follow up study after laser coagulation of placental anastomoses. Endoscopic laser coagulation offers a causal treatment option by interrupting the intertwin blood flow ${ }^{10}$ resulting in normalisation of the right ventricular volume load in the recipient. ${ }^{12}{ }^{13}$ In contrast, amnioreduction reduces amniotic fluid volume and pressure but not the underlying cause of TTTS. After serial amniocentesis, cardiac dysfunction progresses with advancing gestational age, increasing the risk of more severe postnatal cardiac disease. ${ }^{5-8}$ Therefore, fewer fetuses treated with laser coagulation would be expected to develop structural heart disease. Despite successful laser coagulation, we found congenital heart disease and pulmonary stenosis to be as common as after serial amniocentesis. ${ }^{9}$ This suggests that, as previously discussed, factors in very early pregnancy may lead to congenital heart disease and the development of right ventricular outflow tract obstruction in the recipient.

Gardiner $e a^{14}$ showed that abnormal fetal haemodynamic loading is associated with increased arterial wall stiffness in childhood in the donor after serial amniocenteses. Mid trimester laser treatment did not prevent discordant intertwin arterial wall stiffness but did alter the pattern with increased pulse wave velocity in the heavier twin. Further follow up studies-including the possible effect on long term cardiovascular performance-are needed.

\section{Study limitations}

After prenatal laser coagulation, additional follow up data on serial echocardiographic assessments were not available for all fetuses because local referring units followed up the patients. Serial measurement of pulmonary valve diameter and Doppler velocities of the outflow tract is crucial for detecting emerging pulmonary stenosis in the fetus.

Since the start of this prospective study, additional parameters obtained from tissue Doppler imaging have been developed to describe systolic and diastolic function in more detail. Therefore, further studies of this new technology may be necessary.

\section{Conclusion}

This study provides evidence of normalisation of cardiac function in fetuses with severe TTTS even after severe prenatal haemodynamic overload after intrauterine laser coagulation. This illustrates the remarkable adaptability of the developing heart and shows that cardiac hypertrophy and failure regress once the underlying cause has been removed. However, the increased prevalence of pulmonary stenosis in recipients justifies the need for prenatal and postnatal echocardiographic surveillance.

\section{ACKNOWLEDGEMENTS}

We thank Dr M Knapp, PhD, Institute for Medical Biometry, Informatics, and Epidemiology, University of Bonn, Bonn, Germany, for his support in statistical analysis of the study results.

\section{Authors' affiliations}

U Herberg, W Gross, J Brever, Division of Paediatric Cardiology, University of Bonn, Bonn, Germany

P Bartmann, C S Banek, Division of Neonatology, University of Bonn, Bonn, Germany

K Hecher, Department of Obstetrics and Fetal Medicine, University Clinic Hamburg-Eppendorf, Hamburg, Germany

Competing interests statement: The authors have no competing interests to declare.

\section{REFERENCES}

1 Dickinson JE, Evans SF. Obstetric and perinatal outcomes from the Australian and New Zealand twin-twin transfusion syndrome registry. Am J Obstet Gynecol 2000;182:706-12.

2 Duncan KR, Denbow ML, Fisk NM. The aetiology and management of twintwin transfusion syndrome. Prenat Diagn 1997;17:1227-36.

3 Machin G, Still K, Lalani T. Correlations of placental vascular anatomy and clinical outcomes in 69 monochorionic twin pregnancies. Am J Med Genet 1996;61:229-36.

4 De Lia J, Fisk N, Hecher K, et al. Twin-to-twin transfusion syndrome: debates on the etiology, natural history and management. Ultrasound Obstet Gynecol 2000;16:210-3.

5 Zosmer N, Bajoria R, Weiner E, et al. Clinical and echographic features of in utero cardiac dysfunction in the recipient twin in twin-twin transfusion syndrome. Br Heart J 1994;72:74-9.

6 Gembruch U, Bald R, Fahnenstich $\mathrm{H}$, et al. Echocardiographic function in cases of twin-twin transfusion syndrome [abstract]. Ultrasound Obstet Gynecol 1994;4:S183.

7 Fesslova V, Villa L, Nava S, et al. Fetal and neonatal echocardiographic findings in twin-twin transfusion syndrome. Am J Obstet Gynecol 1998; 179:1056-62.

8 Simpson LL, Marx GR, Elkadry EA, et al. Cardiac dysfunction in twin-twin transfusion syndrome: a prospective, longitudinal study. Obstet Gynecol 1998;92:557-62.

9 Karatza AA, Wolfenden JL, Taylor MJ, et al. Influence of twin-twin transfusion syndrome on fetal cardiovascular structure and function: prospective case-control study of 136 monochorionic twin pregnancies. Heart 2002;88:271-7.

10 Hecher K, Plath $\mathrm{H}$, Bregenzer T, et al. Endoscopic laser surgery versus serial amniocenteses in the treatment of severe twin-twin transfusion syndrome. Am J Obstet Gynecol 1999;180:717-24.

11 Hecher K, Diehl W, Zikulnig L, et al. Endoscopic laser coagulation of placental anastomoses in 200 pregnancies with severe mid-trimester twin-to-twin transfusion syndrome. Eur J Obstet Gynecol Reprod Biol 2000;92:135-9.

12 Zikulnig L, Hecher K, Bregenzer T, et al. Prognostic factors in severe twin-twin transfusion syndrome treated by endoscopic laser surgery. Ultrasound Obstet Gynecol 1999;14:380-7.

13 Gratacos E, Van Schoubroeck D, Carreras E, et al. Impact of laser coagulation in severe twin-twin transfusion syndrome on fetal Doppler indices and venous blood flow volume. Ultrasound Obstet Gynecol 2002;20:125-30.

14 Gardiner HM, Taylor MJ, Karatza A, et al. Twin-twin transfusion syndrome: the influence of intrauterine laser photocoagulation on arterial distensibility in childhood. Circulation 2003;107:1906-11. 
15 Banek CS, Hecher K, Hackeloer BJ, et al. Long-term neurodevelopmental outcome after intrauterine laser treatment for severe twin-twin transfusion syndrome. Am J Obstet Gynecol 2003;188:876-80.

16 National High Blood Pressure Education Program. Update on the 1987 task force report on high blood pressure in children and adolescents: a working group report from the National High Blood Pressure Education Program. National High Blood Pressure Education Program working group on hypertension control in children and adolescents. Pediatrics 1996;98:649-58.

17 Kampmann C, Wiethoff CM, Wenzel A, et al. Normal values of M-mode echocardiographic measurements of more than 2000 healthy infants and children in central Europe. Heart 2000;83:667-72

18 Quinones MA, Otto CM, Stoddard M, et al. Recommendations for quantification of Doppler echocardiography: a report from the Doppler quantification task force of the nomenclature and standards committee of the American Society of Echocardiography. J Am Soc Echocardiogr 2002;15:167-84.

19 Riggs TW, Snider AR. Respiratory influence on right and left ventricular diastolic function in normal children. Am J Cardiol 1989;63:858-61.

20 Harada K, Suzuki T, Tamura M, et al. Role of age on transmitral flow velocity patterns in assessing left ventricular diastolic function in normal infants and children. Am J Cardiol 1995;76:530-2.

21 Harada K, Suzuki T, Tamura M, et al. Effect of aging from infancy to childhood on flow velocity patterns of pulmonary vein by Doppler echocardiography. Am J Cardiol 1996:77:221-4.

22 O'Leary PW, Durongpisitkul K, Cordes TM, et al. Diastolic ventricular function in children: a Doppler echocardiographic study establishing normal values and predictors of increased ventricular end-diastolic pressure. Mayo Clin Proc 1998:73:616-28

23 Abdurrahman L, Hoit BD, Baneriee A, et al. Pulmonary venous flow Dopple velocities in children. J Am Soc Echocardiogr 1998;11:132-7.

24 Zhendong $Y$. Effects of age and respiration on right ventricular diastolic filling patterns in normal children. Pediatr Cardiol 1998;19:218-20.

25 Okada $Y$, Ono S, Inove Y, et al. Doppler echocardiographic evaluation of right ventricular diastolic function in children. Pediatr Cardiol 2000;21:358-62.
26 McMahon CJ, Feltes TF, Fraley JK, et al. Natural history of growth of secundum atrial septal defects and implications for transcatheter closure. Heart 2002;87:256-9

27 Fisher DC, Fisher EA, Budd JH, et al. The incidence of patent foramen ovale in 1,000 consecutive patients. A contrast transesophageal echocardiography study. Chest 1995;107:1504-9.

28 Ferencz C, Rubin JD, Loffredo CA, et al. Epidemiology of congenital heart disease: the Baltimore-Washington infant study 1981-1989. Mt Kisko: Futura Publishing, 1993

29 Wren C, Richmond S, Donaldson L. Temporal variability in birth prevalence of cardiovascular malformations. Heart 2000;83:414-9.

30 Lougheed J, Sinclair BG, Fung Kee Fung K, et al. Acquired right ventricular outflow tract obstruction in the recipient twin in twin-twin transfusion syndrome. J Am Coll Cardiol 2001:38:1533-8.

31 Bajoria R, Ward S, Chatteriee R. Natriuretic peptides in the pathogenesis of cardiac dysfunction in the recipient fetus of twin-twin transfusion syndrome. Am J Obstet Gynecol 2002;186:121-7.

32 Nishimura RA, Abel MD, Hatle LK, et al. Assessment of diastolic function of the heart: background and current applications of Doppler echocardiography. Part II. Clinical studies. Mayo Clin Proc 1989:64:181-204.

33 Vermilion RP, Snider AR, Bengur AR, et al. Long-term assessment of right ventricular diastolic filling in patients with pulmonic valve stenosis successfully treated in childhood. Am J Cardiol 1991;686:48-52.

34 Burn J Corney G. Congenital heart defects and twinning. Acta Genet Med Gemellol (Roma) 1984;33:61-9.

35 MacGregor AJ, Snieder H, Schork NJ, et al. Twins: novel uses to study complex traits and genetic diseases. Trends Genet 2000;16:131-4.

36 Lewi L, Van Schoubroeck D, Gratacos E, et al. Monochorionic diamniotic twins: complications and management options. Curr Opin Obstet Gynecol 2003:15: 177-94

37 Yagel S, Weissman A, Rotstein Z, et al. Congenital heart defects: natural course and in utero development. Circulation 1997;96:550-5.

38 Mahieu-Caputo D, Muller F, Joly D, et al. Pathogenesis of twin-twin transfusion syndrome: the renin-angiotensin system hypothesis. Fetal Diagn Ther $2001 ; 16: 241-4$

\section{IMAGES IN CARDIOLOGY}

\section{An unusual cause of chest pain and ST segment elevation}

A 22 year old naval serviceman presented with a six day history of retrosternal chest pain and ST segment elevation on the ECG. The chest pain was not typically ischaemic, nor did the patient have risk factors for coronary disease. The admission ECG (panel A) showed sinus bradycardia, right axis deviation, incomplete right bundle branch block, clockwise rotation, and ST segment elevation in the inferolateral leads. Clinical examination was unremarkable and bedside echocardiography revealed normal left ventricular size and function. A decision was therefore made not to proceed to coronary angiography and the patient was admitted to the ward.

Initial laboratory investigations revealed no evidence of an inflammatory process and markers of myocardial necrosis were negative. The opinion of a radiologist was sought as the plain chest radiograph showed leftward displacement of the cardiac silhouette with a rim of lucency between the inferior border of the heart and the left hemidiaphragm (panel $B$ ), raising the suspicion of congenital absence of the pericardium. This diagnosis was subsequently confirmed by ECG gated computed tomographic (CT) scan of the heart with intravenous contrast (panel C), that demonstrated complete absence of the pericardium around the heart with the coronary arteries lying freely within the epicardial fat. Coronary artery patency was confirmed at angiography.

Complete congenital absence of the pericardium is rare but well described in the literature. However, ST segment elevation has not been reported previously. It should thus be considered in young patients
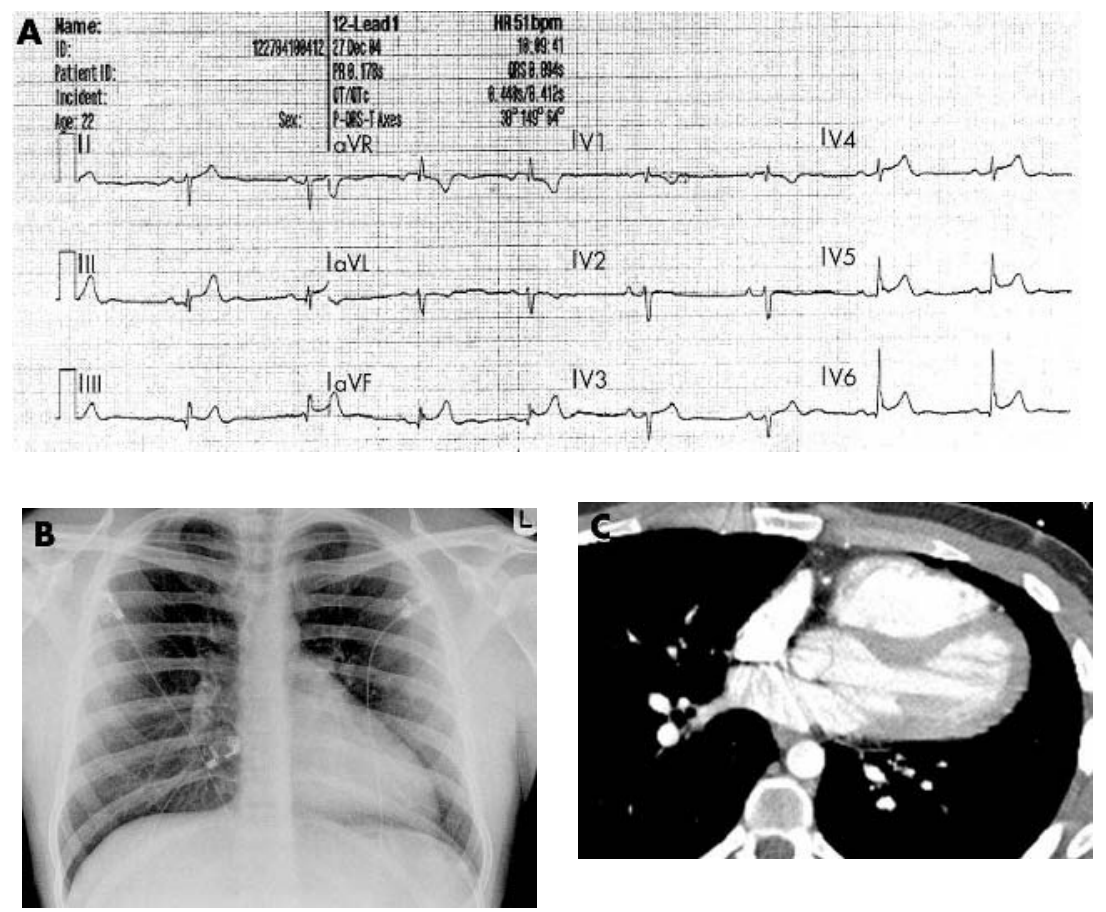

presenting with chest pain. The chest radiograph is suggestive but confirmation usually requires $\mathrm{CT}$ or magnetic resonance imaging.

\section{A P Asherson \\ C D J Ilsley \\ T K Mittal}

a.asherson@rbh.nthames.nhs.uk 